\title{
RESUMING ELECTIVE ONCOLOGIC SURGERY AFTER COVID-19 OUTBREAK: WHAT PRECAUTIONS SHOULD BE TAKEN?
}

\author{
Anass M. Majbar, Amine Benkabbou, Raouf Mohsine, Amine Souadka \\ Digestive oncological surgery department, National Institute of Oncology, Rabat, Morocco. \\ Surgery Department, Mohammed Vth University in Rabat, Morocco.
}

\section{ABSTRACT}

The COVID 19 outbreak has caused the cancellation of most elective oncological surgery around the world to limit the risk of virus dissemination. As we are exiting the crisis, surgical teams will face strong challenges while resuming normal elective surgery. The accumulation of cases will have to be managed by defining strong selection criteria, taking into account the patient and the disease conditions. In order to reduce the risk of infection, nonCOVID patients should be treated in dedicated non-COVID areas, preferably in separate buildings or hospitals. Departments, units and operative theaters should put in place rigorous actions and protocols to protect the patient and healthcare workers. Adequate protective equipment must be readily available for healthcare workers and patients. Finally, teams should keep an adaptive mindset by preparing strategies to maintain surgical activity in case of repeated COVID 19 waves.

Keywords: COVID 19; Guidelines; Lockdown ease; Surgical oncology.

Corresponding Author:

Dr Anass M. Majbar, MD.

Affiliation: Digestive oncological surgery department, National Institute of Oncology. Rabat, Morocco.

E-mail: anass.majbar@um5s.net.ma

Tel.: + (212) 668846573

Copyright (C) 2012- 2020 Dr Anass M. Majbar. This is an open access article published under Creative Commons Attribution -Non Commercial- No Derives 4.0 International Public License (CC BY-NC-ND). This license allows others to download the articles and share them with others as long as they credit you, but they can't change them in any way or use them commercially.

doi: $10.46327 /$ msrjg.1.000000000000161

*****Published in June 02, 2020.

doi url: https://doi.org/10.46327/msrjg.1.000000000000161

\section{INTRODUCTION}

The Coronavirus disease 2019 (COVID-19) pandemic has caused a global health crisis, the short and long term consequences of which are yet to be assessed. In order to reduce the risk of contagion, the majority of countries have opted for a general containment of the population, thus reducing all the activities of the countries [1]. On the health front, there has been a significant reduction in usual medical activity for two main reasons. First, the rearrangement of departments and staff was necessary to manage the large flow of patients with COVID19 , resulting in a significant reduction in the number of beds and nursing staff in clinical departments [2]. And second, all scientific societies have recommended the cancellation and postponement of any non-urgent, postponable clinical activity [3]. These measures have resulted in a significant reduction in surgical activity worldwide [4]. A recent study by the COVIDSurg Collaborative estimated that the total cancellation rate over 12 weeks would be $25.4 \%$ for oncological surgery and that it would take a median period of 45 weeks to purge this delay due to the pandemic.[5].

Now that the epidemic seems to be over and many countries have started deconfinement, medical activity will gradually resume. However, the risk of infection still exists and the possibility of an increase in cases of infection cannot be ruled out.[6, 7]. In surgery in 
particular, the invasive nature of procedures increases the risk of contagion among healthcare personnel. In addition, cancer patients are fragile during the perioperative period and COVID-19 infection could have serious consequences compared to the general population.[8, 9]. Therefore, specific precautionary measures will be necessary in order to reduce the risk of contagion and to protect patients and medical personnel [6].

There are only a few recommendations from scientific societies for resuming normal activity after COVID 19 pandemic [10, 11]. There will certainly be a heavy burden on hospitals to catch up with cancellations and the need to manage patients with progressing cancers [11]. Care teams need to properly prepare and plan for recovery and be able to adapt quickly to any change in the epidemiological situation.

The objective of this article is to propose, through a literature review, expert opinions and guidelines, a series of practical general measures intended for surgical services in order to manage with maximum safety the period of resumption of activity of normal oncological surgery.

\section{GENERAL CONSIDERATIONS}

During the Covid19 pandemic, all scientific societies concentrated on making urgent recommendations for prioritizing surgical activity [12]:

1-Consider other alternatives to surgery (imaging, markers), to assess resectability, locoregional as well as lymph node assessment

2-favor as much as possible a neoadjuvant waiting treatment (Chemotherapy, radiochemotherapy, hormone therapy) and prolongation of waiting intervals after treatment in order to postpone the surgery as much as possible.

Even if the pandemic is receding worldwide and many countries have initiated deconfinement, the risk of contagion is not null. Resumption of surgical activity must be done with the utmost precautions in order to reduce the risk of infection for nursing staff and patients [6].

Due to the reduction in surgical activity for several weeks, pressure on surgical departments is expected to be significant due to the longer waiting lists. The particularity of oncological surgery is that it requires both specialized human resources, advanced resources (physical or material), resuscitation beds but above all dependent on the availability of blood. Surgical teams will have to take up ethical challenges in the face of the scarcity of resources and the shortage of major oncological surgery acts compared to demand. This will require establishing difficult prioritization criteria since it will not be possible to increase surgical activity, while at the same time guaranteeing optimal safety rules. These selection criteria must take into consideration the patient's condition, the nature of the disease and the working environment of each department or hospital.

To reduce the risk of infection, it is important to make sure that the patient or caregiver is not infected or at risk of infection. This will impose strict measures of preparation and reception of patients and perioperative management and also the availability in sufficient quantities of specific protective equipment.

Healthcare systems and healthcare workers, as exhausted as they are, will have to work harder to reduce the impact on cancer patients.

It is important to take into account that the pandemic situation is changing from day to day and that these recommendations are based on our current knowledge, and could become inappropriate if the health situation changes. Each hospital and health system should consider their unique situation in terms of COVID-19 prevalence, staffing capabilities, personal protection equipment supply, and so on when determining how and when to implement these recommendations [13]. 


\section{CRITERIA FOR RESUMPTION OF NORMAL SURGICAL ACTIVITY}

Before resuming normal activity, some important points should be taken into consideration. The resumption of elective surgery can only be done in a context where the epidemic has been controlled and the number of cases is in continuous reduction [6].

Healthcare workers, surgical departments and operating theaters were used as resources in the care of COVID-19 patients during the crisis. Care should therefore be taken to ensure that healthcare personnel are not infected by COVID-19 to avoid the risk of contagion for patients and caregivers. Ideally, all staff should be tested negatively before recovery $[4,6]$. The search for antibodies and antigens to COVID19 by PCR can be done to assess contagion and exposure to the virus. Rapid test kits for antibodies are not recommended in surgical practice due to their variable results $[14,15]$. However, this logical measure will not be applicable in all countries, especially in lowmiddle income countries, where the possibility of testing could be limited. In this case, a confinement period of at least 15 days must be respected by the personnel who participated in the treatment of infected patients, and only asymptomatic people will be authorized to return to work.[16].

Although the pandemic is on the decline, there are still patients who are hospitalized or who will be hospitalized for the management of COVID-19. In addition, it is likely to receive positive patients, requiring urgent surgery. In both cases, it is important to avoid contact between healthy patients and positive or suspect patients. Clear separation of pathways must be implemented and strictly observed, preferably by placing healthy patients and patients with COVID-19 in different buildings or hospitals. [4, 6, 17, 18].

In order to ensure a safe transition to normal activity, a communication strategy intended for nursing staff must be put in place, explaining the organizational changes and the protective measures to be observed.[6]. Education and support for healthcare personnel to accept and adhere to the new post COVID-19 protocols is necessary to prevent the risk of viral transmission [4].

The availability of a sufficient quantity of protective equipment is also an essential condition for resuming normal elective activity.[6]. Authors have advised the availability of sufficient stock for at least 30 days, with efficient supply chains [4].

Finally, strong administrative support is mandatory during this period. Strong measures, such as the reorganization of pathways, the sanctuarization of COVID-19 services, screening of healthcare staff, and the availability of a sufficient quantity of protective equipment, cannot succeed without collaboration between the various hospital departments and strong institutional support, both from the hospital management and the authorities.

\section{Patient selection}

The current crisis has led to the postponement of a large part of the elective interventions for many cancer patients for at least two months. These patients are expected to return to the hospital, adding to the flow of new patients requiring oncological surgery. Delays in treatment will therefore persist and patients will present with more advanced or complicated cancers. Therefore, there will be significant pressure on surgical services during recovery, but their capacities will not be maximal from the start, and it will be necessary to establish strict prioritization criteria [11]. The main criteria reported in the literature are COVID-19 infection, the patient's general condition and the operative indication.

Patients with positive or strongly suspected COVID-19 infection should be postponed until recovery $[6,13]$. The healing criteria are defined according to local regulations. CDC defines healing as absence of symptoms for at least 14 
days [13, 14], whereas in Morocco, two negative tests separated by at least 24 hours are necessary [19].

Because of the delays in treatment, patients will have a progression of the tumor which could change the therapeutic strategy. A reassessment of the disease by suitable paraclinical examinations should quickly be carried out to assess the new oncological situation and propose the appropriate treatments.[11].

For non-infected or cured patients, priority will be decided based on the patient's condition and the oncological status of the disease. Symptomatic patients who may become unresectable if surgery is postponed, and those who have no other treatment alternatives should be prioritized [20, 21].

Some authors have proposed to postpone surgery in elderly or obese patients or with serious cardiopulmonary or immunocompromised co-morbidities, since they have a higher risk of serious forms in the event of COVID-19 infection [13, 22]. These recommendations are relative in cancer patients due to the prognosis due to the disease.

In all cases, these prioritization rules must be decided locally and the decisions must be made by a multidisciplinary team, including surgeons, oncologists, radiotherapists, anesthesiologists, nurses and any other healthcare professional involved in the care of patients.

\section{Patient assessment}

During the entire patient pathway, the challenge will be to reduce the risk of contagion for patients and caregivers. This requires strict monitoring of patients in order to rapidly detect suspected cases of COVID19 infection [23, 24].

Before hospitalization, a detailed discussion with the patient, preferably by telephone or videoconference, will make it possible to look for any symptoms of COVID19 infection or exposure to an infected patient [6]. This conversation will explain in detail the specific measures taken in care facilities during this period, the specific risks and educate patients on the rules to be observed during hospitalization $[13,25,26]$. This will also make it possible to psychologically prepare the patient, to obtain his collaboration and his consent to undergo the surgery under these conditions [6].

In addition, several authors have proposed to systematically perform a virus screening by PCR in all patients [4, 13, 14, 27], before surgery. Because of the moderate sensitivity of the PCR, the association with a chest CT was recommended [6, 28]. Known features of COVID-19 on lung CT-scan include bilateral, multilobular, ground-glass opacification with a peripheral or posterior distribution [29]. Some patients with a negative PCR may display abnormalities on chest CT-scan [30]. The combination of PCR and chest CT-scan is associated with better sensitivity (92\%) compared to PCR alone (78\%), or chest CTscan alone (67\%) [31]. Combination of both was therefore advised by some authors [6]. Since systematic screening by PCR may not be possible in some contexts, it may be performed only in case of a high suspicion of infection or be replaced by a chest CT scan. The presence of suspicious signs on the latter should lead to a postponement of surgery [13]; the use of antigen tests is not recommended due to their variable results [13]. Other explorations have also been proposed such as CRP, LDH, or testing for viruses in the stool $[6,28,32]$, but no specific recommendations could be made about them.

In all cases, these measures must be adapted by the care teams to their own context and any suspicion of COVID-19 infection will lead to a postponement of the intervention until confirmation of the absence of the disease or patient recovery.

\section{Measures in hospital departments}

Upon arrival at the hospital, the patient should be reassessed clinically for suspicious symptoms. In the absence of suspicious signs, 
the patient is admitted and will be made aware of hygiene measures and distanciation from other hospitalized patients. It is preferable that the hospital provides the patient with protective masks and means of hygiene $[13,26]$. During the entire hospitalization, the staff will ensure that the strict hygiene and distancing measures are respected by the patients and will have to be monitored daily for suspicious symptoms [24]. It is strongly advised that patients wear a mask during the entire hospitalization period [33-35]

In order to limit the risk of infection, certain measures must be put in place in the departments. First, hospitalizations must only be in individual rooms. Even if this will lead to a limitation of the bed capacities, this measure cannot be reasonably lifted until after the declaration of the end of the pandemic by the authorities [13]. Second, visits should be kept to a minimum [13]. Visitors must follow the same suspicious case detection procedure described above and observe strict rules such as wearing a mask and distancing. For example, in our department, visits are limited to one patient per day per patient, and when strictly necessary. At the same time, families were given a phone number to get informed and reduce the need to come to the hospital.

For healthcare personnel, the protective measures must be strictly observed, and which will vary depending on the interactions they will have with patients. Wearing N95 or PPF2 masks and eye protection is strongly advised for any interaction with patients.

\section{OPERATING THEATER SPECIFIC MEASURES}

During this post-crisis period, the objective of oncological surgery will be to offer adequate treatment to cancer patients in an atmosphere of maximum safety while protecting the personnel in the operating room. Any patient entering the operating room should be considered as a possible asymptomatic carrier and cared for by asymptomatic caregivers.

\section{a)- Patient protection in the operating room}

\section{Systematic staff test}

In Morocco, considerable effort has been made to ensure the protection of patients treated in hospitals that have been in contact with COVID 19 through daily surveillance and the reporting and treatment of all cases of healthcare personnel infected with the virus [2]. The day when serological tests will be generalized and available on a large scale, healthcare personnel, especially those in the operating room, should have priority over testing in order to minimize the risk of contaminating patients in the operating room.[36]

All suspicious or symptomatic people should refrain from being in contact with cancer patients as well as the rest of the nursing staff, until the return of negative test results and disappearance of symptoms.

\section{Cleaning measures}

Viral transmission is mainly through saliva droplets, which justifies measures of distancing and hand hygiene, but also probably by orofecal contact [32]. That said, the virus can remain on the surfaces, a priori several hours, reason why after any surgical intervention and before the reception of a new patient, the medical and paramedical and cleaning staff will have to work to apply draconian hygiene measures in accordance with national and local recommendations (according to the nosocomial infection control committee (CLIN)) of each hospital.[37] It is therefore essential to reorganize the operating rooms in order to limit the number of instruments and flat surfaces to be cleaned to a minimum, and favor the use of several operating rooms in turn to facilitate cleaning.[38] 


\section{b)- Protection of operating theater personnel} [13]

\section{In the operating theater}

The sanctuarization measures for Covid-free areas necessary for resuming activity, take all their importance in the operating and resuscitation areas.[24]. It is essential to preserve CoVID free spaces but above all to keep a possible pathway for positive COVID 19 to manage patients requiring immediate treatment of their oncological surgical emergency. An experience that succeeded in Singapore during the peak of the epidemic and which inspired several countries.[39, 40]

There is a debate concerning the value of using negative pressure rooms (turn off positive pressure) at least in COVID-19 operating theaters,[36] that said, this may increase the risk of postoperative infection in patients operated on under these conditions.

\section{Measures for operating room staff}

In order to protect the operating room staff,[1] all scientific societies $[3,8,17,41]$ converge on the fact that it is imperative to:

- Communicate and make staff aware of the importance of applying and respecting general hygiene and distancing measures

- To limit the number of personnel in the operation theater to the strict minimum.

- To reduce the opening and closing of doors and to reduce traffic between the different areas of the operating theater.

- To respect the wearing of protective equipment:

*FFP2 mask (possibly a visor if available) for anesthesia staff and staff included in the operating surgical field.

\footnotetext{
*Surgical masks for staff in operating rooms

*Gloves (with hygienic rules)
}

- Reducing the use of energy devices and favoring the use of bipolar coagulation over monopolar.

- Ensuring the proper functioning of the suction system

- Ensuring adequate disposal of contaminated waste at the end of the procedure, follows the rules established by the hygiene teams and infectiologists.

\section{Anesthesia considerations}

The procedure represents the most at risk moment of contamination by COVID 19 virus during a surgical intervention [42] [43]. Particular attention must therefore be paid to the risk linked to aerosols and droplets:

- The anesthesia team must prepare its action plan with a clear assignment of the respective tasks, and senior management of the airways must be carried out by the most experienced senior.

- Tracheal intubation is preferable to the laryngeal mask and the use of videolaryngoscopy can be proposed as a first intention if available.

- After the procedure, all material not protected by a hydrophobic filter used for ventilation and the material for intubation is discarded or disinfected with a standard disinfectant detergent.

\section{Laparoscopy or laparotomy?}

Today, the laparoscopic approach has clearly demonstrated its advantages in the treatment of cancer patients by reducing the operating risk, the length of stay and the use of analgesics postoperatively. However in a pandemic situation, this approach raised a controversy around the risk of aerosolization of the virus in the operating field. [44] With only a few in Vitro data, some scientific societies have banned this approach in favor of an open approach, at a time 
when others continue to advocate it on the condition of doubling vigilance in order to protect personnel of care by: [45][41][44]

- limiting the nursing staff in the operating room

-favoring low pneumoperitoneum pressures

-using the balloon trocars, to limit the leaks

-using smoke aspirators fitted with filters (HEPA) or at least aspiration of all the pneumoperitoneum in order to avoid any externalization of blown air in the operating field.

Pending more conclusive studies, the staff must be made aware of the potential operating risk without depriving cancer patients of the advantages of this minimally invasive approach.

\section{POST-OPERATIVE MANAGEMENT}

In this Covid 19 outbreak, resuming oncological surgery needs specific concerns of postoperative care. Due to all difficulties reaching in-hospital consultation, telehealth represents one of the most reported recommendation of evaluating surgical patients postoperatively. Postoperatively, telemedicine through teleconsultation offers a variety of modalities easily accessible by patients and surgeons. Video conferences and pictures were taken on a smartphone are effective for followups, including the detection of post-operative complications and even saves patients time.[46, 47]

\section{CONCLUSION}

Teams will face difficult times during the transition to normal activities. Good planification, preparation and collaboration are necessary to manage the burden of delayed surgical cases, more advanced cancer in addition to new patients. Surgical priorities should be defined and hard decisions will have to be made. Rigorous protocols should be maintained to protect patients and healthcare workers from the risk of infection. Teams should also anticipate the possibility of additional COVID 19 waves and prepare adaptive measures to maintain oncologic surgical activity in non-COVID 19 units.

\section{DECLARATION OF INTERESTS: None}

\section{REFERENCES:}

1. Souadka A, Benkabbou A, Al Ahmadi B, Boutayeb S, Majbar MA. Preparing African anticancer centres in the COVID-19 outbreak. The lancet oncology. 2020. p. e237.

2. Souadka A, Essangri H, Benkabbou A, Amrani L, Majbar MA. COVID-19 and Healthcare worker's families: behind the scenes of frontline response. EClinical Medicine. 2020; 100373.

3. COVID-19: Guidance for Triage of NonEmergent Surgical Procedures. In: American College of Surgeons [Internet]. [cited 25 May 2020]. Available: https://www.facs.org/covid19/clinical-guidance/triage

4. Iyengar KP, Jain VK, Vaish A, Vaishya R, Maini L, Lal H. Post COVID-19: Planning strategies to resume orthopaedic surgery challenges and considerations. J Clin Orthop Trauma. 2020. doi:10.1016/j.jcot.2020.04.028

5. CovidSurg Collaborative, Nepogodiev D, Bhangu A. Elective surgery cancellations due to the COVID-19 pandemic: global predictive modelling to inform surgical recovery plans. Br J Surg. 2020. doi:10.1002/bjs.11746

6. Mouton C, Hirschmann MT, Ollivier M, Seil R, Menetrey J. COVID-19 - ESSKA guidelines and recommendations for resuming elective surgery. J Exp Orthop. 2020;7: 28.

7. Prem K, Liu Y, Russell TW, Kucharski AJ, Eggo RM, Davies N, et al. The effect of control strategies to reduce social mixing on outcomes of the COVID-19 epidemic in Wuhan, China: a modelling study. Lancet Public Health. 2020; 5: e261-e270. 
8. Berardi G, Levi Sandri GB, Colasanti M, Ettorre GM. Readaptation of surgical practice during COVID-19 outbreak: what has been done, what is missing and what to expect. $\mathrm{Br} \mathrm{J}$ Surg. 2020. doi:10.1002/bjs. 11698

9. Lei S, Jiang F, Su W, Chen C, Chen J, Mei W, et al. Clinical characteristics and outcomes of patients undergoing surgeries during the incubation period of COVID-19 infection. EClinical Medicine. 2020; 100331.

10. Pandemic Influenza Preparedness and Response: A WHO Guidance Document. Geneva: World Health Organization; 2013.

11. Brindle ME, Doherty G, Lillemoe K, Gawande A. Approaching Surgical Triage During the COVID-19 Pandemic. Ann Surg. 2020. doi:10.1097/SLA.0000000000003992

12. Burki TK. Cancer guidelines during the COVID-19 pandemic. The Lancet Oncology. 2020. pp. 629-630. doi:10.1016/s14702045(20)30217-5

13. Parvizi J, Gehrke T, Krueger CA, Chisari E, Citak M, Van Onsem S, et al. Resuming Elective Orthopaedic Surgery During the COVID-19 Pandemic. The Journal of Bone and Joint Surgery. 2020. p. 1. doi:10.2106/jbjs.20.00844

14. CDC. Coronavirus Disease 2019 (COVID19). In: Centers for Disease Control and Prevention [Internet]. 19 May 2020 [cited 24 May 2020]. Available: https://www.cdc.gov/coronavirus/2019$\mathrm{nCoV} / \mathrm{hcp} /$ clinical-criteria.html

15. Mallapaty S. Will antibody tests for the coronavirus really change everything? Nature. 2020; 580: 571-572.

16. National coronavirus response: A road map to reopening | American Enterprise Institute AEI. In: American Enterprise Institute - AEI [Internet]. [cited 24 May 2020]. Available: https://www.aei.org/researchproducts/report/national-coronavirusresponse-a-road-map-to-reopening/

17. Low TY, So JBY, Madhavan KK, Hartman M. Restructuring the surgical service during the COVID-19 pandemic: experience from a tertiary institution in Singapore. Br J Surg. 2020. doi:10.1002/bjs.11701

18. Al-Jabir A, Kerwan A, Nicola M, Alsafi Z, Khan M, Sohrabi C, et al. Impact of the coronavirus (COVID-19) pandemic on surgical practice - Part 2 (surgical prioritisation). Int J Surg. 2020. doi:10.1016/j.ijsu.2020.05.002

19. البو ابة الرسمية لفيروس كورونا بالمغرب ] cited 26 May 2020].

Available: http://www.covidmaroc.ma/Pages/Profession nelSanteAR.aspx

20. Downs JS, Wilkinson MJ, Gyorki DE, Speakman D. Providing cancer surgery in the COVID-19 crisis. Br J Surg. 2020. doi:10.1002/bjs.11693

21. Qadan M, Hong TS, Tanabe KK, Ryan DP, Lillemoe KD. A Multidisciplinary Team Approach for Triage of Elective Cancer Surgery at the Massachusetts General Hospital during the Novel Coronavirus COVID-19 Outbreak. Ann Surg. 2020. doi:10.1097/SLA.0000000000003963

22. Public Health England. Guidance on shielding and protecting people who are clinically extremely vulnerable from COVID-19. 2020 [cited 24 May 2020]. Available: https://www.gov.uk/government/publications/ guidance-on-shielding-and-protectingextremely-vulnerable-persons-from-covid19/guidance-on-shielding-and-protectingextremely-vulnerable-persons-from-covid-19

23. Lavoué V, Akladios C, Gladieff L, Classe JM, Lécuru F, Collinet P. Onco-gynecologic surgery in the COVID-19 era: Risks and precautions-A position paper from FRANCOGYN, SCGP, SFCO, and SFOG. J Gynecol Obstet Hum Reprod. 2020; 101787.

24. Ghannam A, Souadka A. Beware of Time Delay and Differential Diagnosis when Screening for Symptoms of COVID-19 in Surgical Cancer Patients. J Am Coll Surg. 2020. doi:10.1016/j.jamcollsurg.2020.04.032

25. UpToDate. [cited 24 May 2020]. Available: https://www.uptodate.com/contents/coronavir us-disease-2019-covid-19-overview-thebasics?topicRef $=127551 \&$ source $=$ see_link 
26. Massey PA, McClary K, Zhang AS, Savoie FH, Barton RS. Orthopaedic Surgical Selection and Inpatient Paradigms during the Coronavirus (COVID-19) Pandemic. J Am Acad Orthop Surg. 2020; 28: 436-450.

27. Péré $\mathrm{H}$ et al. Nasal swab sampling for SARSCoV-2: A convenient alternative in time of nasopharyngeal swab shortage. J Clin Microbiol. 2020. doi:10.1128/JCM.00721-20

28. Rodriguez-Morales AJ et al. Clinical, laboratory and imaging features of COVID19: A systematic review and meta-analysis. Travel Med Infect Dis. 2020; 34: 101623.

29. Salehi S, Abedi A, Balakrishnan S, Gholamrezanezhad A. Coronavirus Disease 2019 (COVID-19): A Systematic Review of Imaging Findings in 919 Patients. AJR Am J Roentgenol. 2020; 1-7.

30. Xie X, Zhong Z, Zhao W, Zheng C, Wang F, Liu J. Chest CT for Typical 2019-nCoV Pneumonia: Relationship to Negative RT-PCR Testing. Radiology. 2020; 200343.

31. Jiang G, Ren X, Liu Y, Chen H, Liu W, Guo $\mathrm{Z}$, et al. Application and optimization of RTPCR in diagnosis of SARS-CoV-2 infection. doi:10.1101/2020.02.25.20027755

32. Y. Cherradi. CoVID-19: a digestive disease! Journal of Medical and Surgical Research JMSR- 2020; 6 (3): 714-721. Doi: 10.46327/msrjg.1.000000000000159. doi url: https://doi.org/10.46327/msrjg.1.0000000000 $\underline{00159}$

33. Klompas M, Morris CA, Sinclair J, Pearson M, Shenoy ES. Universal Masking in Hospitals in the Covid-19 Era. N Engl J Med. 2020; 382: e63.

34. Advani SD, Smith BA, Lewis SS, Anderson DJ, Sexton DJ. Universal masking in hospitals in the COVID-19 era: Is it time to consider shielding? Infect Control Hosp Epidemiol. 2020; 1-2.

35. Guo X, Wang J, Hu D, Wu L, Gu L, Wang Y, et al. Survey of COVID-19 Disease Among Orthopaedic Surgeons in Wuhan, People's Republic of China. J Bone Joint Surg Am. 2020; 102: 847-854.
36. Björn L D, Nigri G, Tinelli A, Lapeña JFF, Espin-Basany E, Macri P, et al. COVID-19: Pandemic surgery guidance. 4open. 2020; 3: 1.

37. Tuech J-J, Gangloff A, Di Fiore F, Michel P, Brigand C, Slim K, et al. Strategy for the practice of digestive and oncological surgery during the Covid-19 epidemic. J Visc Surg. 2020. doi:10.1016/j.jviscsurg.2020.03.008

38. Dexter F, Elhakim M, Loftus RW, Seering MS, Epstein RH. Strategies for daily operating room management of ambulatory surgery centers following resolution of the acute phase of the COVID-19 pandemic. J Clin Anesth. 2020; 64: 109854.

39. Tan K-K, Moran BJ, Solomon MJ. Avoiding collateral mortality in a pandemic - time to change our mindset in surgical oncology. Nature Reviews Clinical Oncology. 2020. doi:10.1038/s41571-020-0383-1

40. National University Cancer Institute of Singapore (NCIS) Workflow Team. A segregated-team model to maintain cancer care during the COVID-19 outbreak at an academic center in Singapore. Ann Oncol. 2020. doi:10.1016/j.annonc.2020.03.306

41. Chadi SA et al. Current Evidence for Minimally Invasive Surgery during the COVID-19 Pandemic and Risk Mitigation Strategies: A Narrative Review. Ann Surg. 2020. doi:10.1097/SLA.0000000000004010

42. Cook TM. Personal protective equipment during the coronavirus disease (COVID) 2019 pandemic - a narrative review. Anaesthesia. 2020. doi:10.1111/anae.15071

43. Herman JA, Urits I, Kaye AD, Urman RD, Viswanath O. COVID-19: Anesthesia Management Recommendations. J. Clin Anesth. 2020; 109840.

44. Francis N, Dort J, Cho E, Feldman L, Keller D, Lim R, et al. SAGES and EAES recommendations for minimally invasive surgery during COVID-19 pandemic. Surg Endosc. 2020; 34: 2327-2331.

45. Emile SH. Should we continue using laparoscopy amid the COVID-19 pandemic? Br J Surg. 2020. doi:10.1002/bjs.11669 


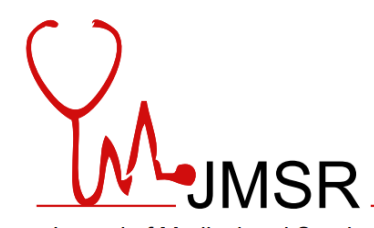

Journal of Medical and Surgical Research

46. Totten AM, Mc Donagh MS, Wagner JH. The Evidence Base for Telehealth: Reassurance in the Face of Rapid Expansion during the COVID-19 Pandemic. 2020. doi:10.23970/ahrqepccovidtelehealth

47. Hakim AA, Kellish AS, Atabek U, Spitz FR, Hong YK. Implications for the use of telehealth in surgical patients during the COVID-19 pandemic. Am J Surg. 2020. doi:10.1016/j.amjsurg.2020.04.026 